\title{
Simulations of helicopter ditching using smoothed particle hydrodynamics*
}

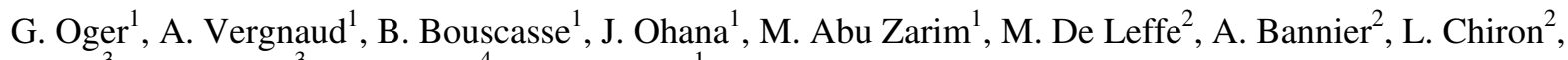 \\ Y. Jus ${ }^{3}$, M. Garnier ${ }^{3}$, S. Halbout ${ }^{4}$, D. Le Touzé ${ }^{1}$ \\ 1. Ecole Centrale de Nantes, LHEEA Laboratory (ECN and CNRS), Nantes, France \\ 2. Nextflow Software, Nantes, France \\ 3. Bureau Veritas Solutions Marine and Offshore, Nantes, France \\ 4. Airbus Helicopters, Marseille, France
}

\begin{abstract}
The present work has been performed in the context of the European H2020 project increased SAfety and Robust certification for ditching of Aircrafts and Helicopters (SARAH) dedicated to improving the safety during aircraft ditching, together with a better understanding of the physics involved during those crucial events. Both numerical and experimental aspects are explored during this project. The present study focuses on the application of the smoothed particle hydrodynamics (SPH) method to the simulation of helicopter ditching, as this method has proved to be particularly adapted to free surface impact cases. Simulations are performed for three different impact configurations, for which the numerical solutions are compared with the experimental results (forces and kinematics) obtained at the wave basin of Ecole Centrale Nantes on a mock-up shape provided by Airbus Helicopters. Elements of sensitivity analysis are also provided when needed, to assess the role of some parameters involved in the helicopter behavior and the fluid pressure forces exerted during the impact.
\end{abstract}

Key words: Helicopter ditching, smoothed particle hydrodynamics (SPH), Free surface impact, wave impact, numerical vs experimental solutions

\section{Introduction}

The European H2020 project SARAH aims at establishing novel holistic, simulation-based approaches to the analysis of ditching in order to increase the safety of aircrafts and helicopters in ditching/floatation situations with validations against dedicated experiments. A better understanding of the various phenomena occurring during such events and enhanced simulation tools are expected thanks to this project. To this aim, experiments were made in partnership with Airbus Helicopters ${ }^{[1]}$, providing reference results for comparison with computational fluid dynamics (CFD) solvers. While both aircraft and helicopters cases are treated in this project, the present work focuses more specifically on the helicopter ditching. During such an event, and because of the high position of the center of gravity (engine and rotor), it is a challenge for the helicopter to avoid to capsize. In order to improve the chances of survival of

\footnotetext{
* Biography: G. Oger (1979-), Male, Ph. D. Research Fellow Corresponding author: G. Oger,

E-mail: guillaume.oger@ec-nantes.fr
}

the occupants, an emergency floatation system (EFS) usually made of inflatable floats is deployed prior to the impact, keeping the helicopter afloat for a sufficiently long duration. Nevertheless, predicting the helicopter and EFS behavior during the impact is not obvious, especially in presence of waves. The regulation $^{[2-3]}$ frames the conditions of impact to be considered by an applicant designing an helicopter dedicated to operate over water area. An envelope of values of key parameters is prescribed: velocities, sea conditions, helicopter attitude... For such complex impact cases, the numerical simulations stand as a pertinent complementary tool to the experiments. Indeed, although experiments provide trustable results, some uncertainties (bias and repeatability) are caused by various factors as the structural deformation of the model backbone, the position of the sensor who may measure also significant inertia forces, or the sensitivity of the impact forces on a free-falling body to some light change of experimental conditions. On the other hand, while numerical simulations do not guarantee a perfect solution, they form a precious tool for providing the global trends in correlation with local effects that cannot be addressed in the experiments. This complementary approach is out- 
lined in the present work.

The experiments presented in the paper were performed in the wave basin facilities of Ecole Centrale Nantes ${ }^{[1]}$. A large set of helicopter impact cases with and without waves were tested. A numerical study is performed with the SPH method, a good candidate for such complex numerical simulations thanks to its meshless and Lagrangian features and its ability to deal with complex geometries and large body motions in interaction with a free surface submitted to strong deformations ${ }^{[4-5]}$. Some simulations of helicopter ditching using the smoothed particle hydrodynamics (SPH) method have already been performed in the past ${ }^{[6-9]}$. Among these works, Xiao et al ${ }^{[9]}$ investigated the ditching in calm water of an helicopter impacting with three different pitch angles highlighting a nose-down nose-up pitching motion. The present paper covers also this aspect, investigating the pitch angle evolution in time and its effects on the resulting flow, hydrodynamic forces and helicopter behavior, with and without waves.

This paper first describes the experimental setup and then presents the SPH scheme with a brief discussion of the functionalities needed to perform such complex simulations. Numerical and experimental results are finally compared, and conclusions are drawn.

\section{Experimental setup}

\subsection{Experimental design}

The helicopter ditching experiments exploited in this study aim at measuring the trajectory evolutions in time as well as the forces exerted on parts of the helicopter structure for a wide range of impact configurations, i.e., for different impact attitudes and velocities on flat water or in waves.

\subsubsection{Rail guiding system}

After some iterations including motorized solutions the final choice has been made to rely on a gravity system similar to what is done for roller coasters (rails+carriage/trolley), as presented in Fig. 1. The facility consists in a guiding system able to launch the helicopter as accurately as possible in various ditching scenarios. Differently from the tests conducted in Ref. [10] for aircraft ditching, the guided rail in this campaign ended in the horizontal plane.

The system is composed of four rails, two externals and two internals. The trolley, equipped with four wheels, is responsible for supporting the helicopter mock-up. The aim is to reproduce helicopter ditching events with conditions equivalent or close to those prescribed in the regulation. The

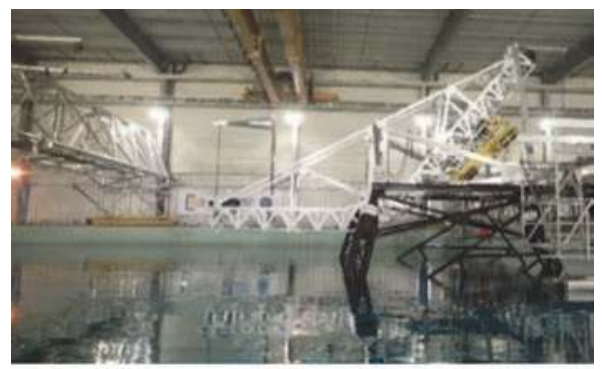

(a)

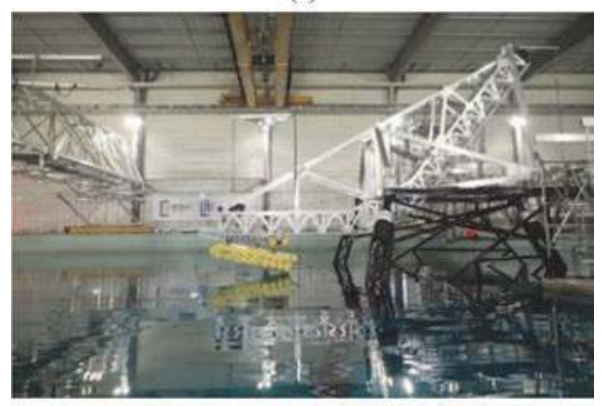

(b)

Fig. 1 (Color online) Rail guiding system in the ECN's wave basin

mock-up is launched at a certain height from the water and ditches after a short free flight. The system must be able to control the velocity of the mock-up. The challenging task is to control a low vertical speed (a free fall of few centimeters) together with a large horizontal velocity. This resulted in a $10 \mathrm{~m}$ long and 5 $\mathrm{m}$ high rail guiding system (excluding the underwater structure). The mock-up was positioned at four different launching heights, inducing a speed during free flight of approximately $8 \mathrm{~m} / \mathrm{s}, 7 \mathrm{~m} / \mathrm{s}, 6 \mathrm{~m} / \mathrm{s}$ and $0.4 \mathrm{~m} / \mathrm{s}$, respectively.

The launching system functions are:

(1) pick up the model and mount it on the system,

(2) launch it at a precise time and accelerate it to an expected speed,

(3) finally drop/let the model in free flight.

\subsubsection{Helicopter mock-up}

The mock-up used in the experiments is $1 / 3.4$ with respect to the full scale helicopter, and is shown in Fig. 2.

It is connected to the trolley through an interface piece responsible for varying the helicopter attitudes (pitch, roll and yaw angles).

As illustrated in Fig. 3, this mock-up is composed of:

(1) a cabin,

(2) four rigid floats,

(3) two sponsons linking the rear floats to the cabin,

(4) girths fixing the floats to the cabin/sponsons (three girths for each float). 


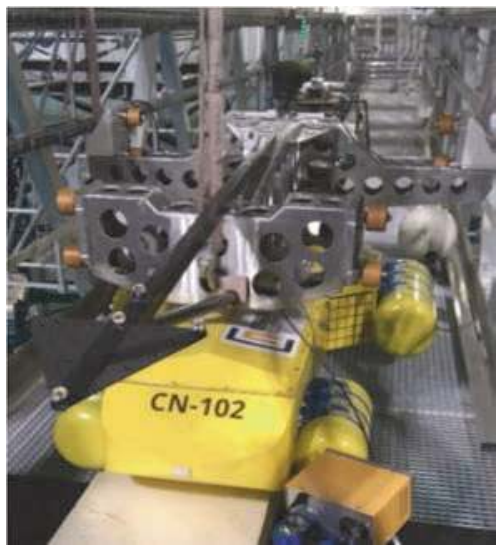

Fig. 2 (Color online) View of the helicopter mock-up and its trolley

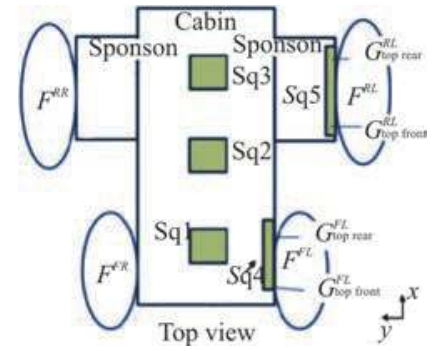

Fig. 3 (Color online) Schematic description and nomenclature of the mock-up components

The four rigid floats are composed of circular cylinders ended with rounded shape tips. Other impact campaigns were performed with inflatable floats, but such configurations are not discussed in the present work to make clearer the comparison with the numerical method results.

Three sensors were placed under the fuselage (see Fig. 4). Those sensors connect three squared plates $(75 \mathrm{~mm} \times 75 \mathrm{~mm}) \mathrm{Sq} 1, \mathrm{Sq} 2$ and $\mathrm{Sq} 3$ to the backbone, and are dedicated to "local" force measurements during the impact. A preliminary SPH analysis conducted with a prototype geometry showed the vertical force peak duration to be of the order of $0.01 \mathrm{~s}$. This induces the choice of Kistler 9227A piezoelectric sensors. Two additional force and moment 6DOF sensors were used for the interfaces float/cabin and float/sponson, named respectively Sq4 and $\mathrm{Sq} 5$ in Fig. 3.

Finally, the trajectories of the mock-up during its impact on water were tracked using an optical system "QUALISYS". Many LEDs were arranged on the mock-up: some were located on the trolley and others on the cabin. Six motion tracking cameras were placed at different positions over the basin in order to maximize the capability to track the LEDs. Three high speed velocity cameras were also included in the system for visualization, including one underwater. Note that the use of such a system was challenging, particularly because of the large sprays immediately occurring after the helicopter impact. Furthermore, an IMU inertial unit SBG ELLIPSE2-E-G5A4_B2 was positioned in the backbone of the mock-up. Roll, pitch and yaw angles were directly obtained from this unit.
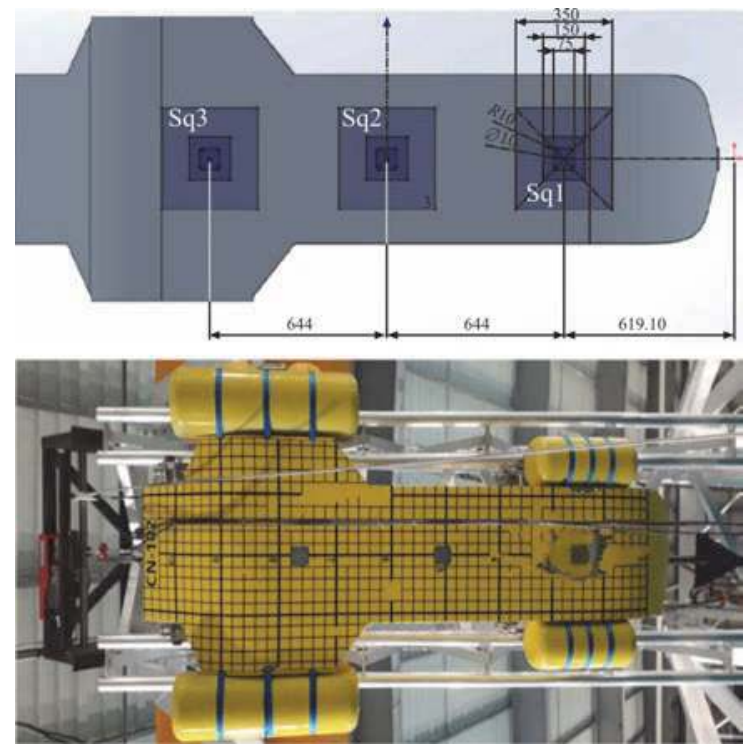

Fig. 4 (Color online) Bottom view of the helicopter mock-up with force sensors Sq1-Sq3

\subsection{Impact conditions studied}

The global frame of reference is given by the axis system $\{O, X, Y, Z\}$, where $Z$ is the vertical (gravity) axis and for which the free surface of calm water is located at $Z=0$. is the direction of the rail, with $X=0$ the beginning of the rail. We also introduce an additional frame of reference $\{O h, X h, Y h, Z h\}$ attached to the helicopter (see Fig. 5) used during the experimental campaign. For all impact cases discussed in the present work, only the pitch angle $\theta$ differs from zero at the initial instant.

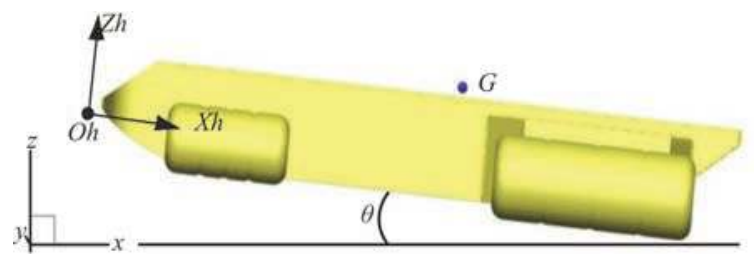

Fig. 5 (Color online) Schematic of the referential system adopted in the experiments. The mock-up is presented here at the impact instant with the conditions of the considered case

Furthermore, the inertial properties of the mock-up with respect to point $G$ are summarized in Table $1, G$ being the Center of Gravity (CoG). 
Table 1 Inertial properties of the helicopter mock-up. Positions are provided in the helicopter frame of reference $\{O h, X h, Y h, Z h\}$

\begin{tabular}{cccccccccc}
$X_{G} / \mathrm{m}$ & $Y_{G} / \mathrm{m}$ & $Z_{G} / \mathrm{m}$ & Mass $/ \mathrm{kg}$ & $J_{x x} / \mathrm{kg} \cdot \mathrm{m}^{2}$ & $J_{y y} / \mathrm{kg} \cdot \mathrm{m}^{2}$ & $J_{z z} / \mathrm{kg} \cdot \mathrm{m}^{2}$ & $J_{x y} / \mathrm{kg} \cdot \mathrm{m}^{2}$ & $J_{x z} / \mathrm{kg} \cdot \mathrm{m}^{2}$ & $J_{y z} / \mathrm{kg} \cdot \mathrm{m}^{2}$ \\
\hline 1.49 & -0.013 & 0.238 & 348.1 & 84.3 & 190.7 & 205.8 & -0.5 & 4.4 & -0.5 \\
\hline
\end{tabular}

\subsection{Experimental uncertainties}

Experimental setup and measurements are affected from various uncertainties listed below that need to be considered when comparing to numerical tests.

Mass, inertia and CoG position of the mock-up. In particular, the longitudinal $\mathrm{CoG}$ position of the mock-up suffers from small uncertainties while this parameter strongly affects the impact conditions.

Before and after impact the mock-up is in free flight with $6 \mathrm{DOF}$. This together with the size of the experiment and the large mass and size of the mock-up affects the repeatability of the kinematics around the impact instant. Determining the exact impact instant is complex, making difficult to evaluate precisely the longitudinal, vertical and angular (pitch) velocities used for the initial condition of the numerical simulations. The analysis of the experimental measurements and the synchronization of the channels acquired by various systems have been improved over time and the initial conditions for the numerical tests (pitch angle and velocity) might be slightly different from those obtained in the finalized version (of the order of $0.1^{\circ} / 0.2^{\circ}$ ). The synchronization is more difficult for the case involving waves, leading to uncertainties related to the crest and helicopter cabin relative positions at the impact instant.

Each sensor measures hydrodynamic and inertial forces on the plate it is holding. At impact the vertical acceleration is very strong, and the inertial effect may be more or less significant.

\section{Numerical scheme and parameters}

\subsection{SPH scheme adopted}

In this impact problem, we assume the Reynolds number to be sufficiently large to neglect the viscous effects, so that the governing system to solve reduces to the Euler equations. Moreover, as a first attempt of comparison between simulations and experiments, the air phase is assumed to play a minor role and is therefore absent from the simulations, treated here in a single-phase approach. On the other hand, this work is based on the weakly-compressible Riemann-based SPH scheme. Note that a null mass flux is imposed, as proposed by Parshikov et al. ${ }^{[11]}$

$$
\begin{aligned}
& \frac{\mathrm{d} \boldsymbol{x}_{i}}{\mathrm{~d} t}=\boldsymbol{v}_{0 i} \\
& \frac{\mathrm{d}\left(\rho_{i} V_{i}\right)}{\mathrm{d} t}=0 \\
& \frac{\mathrm{d} V_{i}}{\mathrm{~d} t}=2 V_{i} \sum_{j \in P} V_{j}\left(\boldsymbol{v}_{E}-\boldsymbol{v}_{i}\right) \cdot \nabla_{i} W_{i j} \\
& \frac{\mathrm{d}\left(\rho_{i} V_{i} \boldsymbol{v}_{i}\right)}{\mathrm{d} t}=-2 V_{i} \sum_{j \in P} V_{j} P_{E} \nabla_{i} W_{i j}+\rho_{i} V_{i} \boldsymbol{g}
\end{aligned}
$$

where $\boldsymbol{x}_{i}, \boldsymbol{v}_{i}, \boldsymbol{v}_{0 i}, V_{i}$ and $\rho_{i}$ refer respectively to the position, the Lagrangian velocity, the particle velocity (including the shifting velocity ${ }^{[12]}$ ), the discrete volume and the density of the particle $i . g$ is the acceleration of gravity and $W$ the kernel function taken here as the Wendland kernel ${ }^{[13]}$. The subscript $E$ refers to the numerical solution of the Riemann problem between particles $i$ and $j$, solved at the location $\boldsymbol{x}_{i j}=\left(\boldsymbol{x}_{i}+\boldsymbol{x}_{j}\right) / 2$. This system is closed using the Cole's equation of state, used throughout the present study. The artificial speed of sound used for water (noted $c_{0}$ hereafter) is chosen so that the Mach number does not exceed 0.1 to stay in the weakly-compressible range. This consideration leaded to use $c_{0}=300 \mathrm{~m} / \mathrm{s}$.

\subsection{Boundary conditions}

As shown in the above figures, the geometry of the helicopter mock-up is rather complex, and it presents convex surfaces and sharp edges. For such a geometry, the classical ghost (mirroring) particle technique is quite unsuitable. The alternative technique used here is a Boundary Integral Method with the "cut-face approach" proposed by Chiron et al. ${ }^{[14]}$. It consists in computing the surface terms in SPH operators while applying the required boundary conditions. Note that these surface terms are directly used to perform the pressure force integration onto the geometry, allowing then the 6DOF motion of the helicopter (considered here as a free rigid body) in coupled interaction with water. 


\subsection{Spatial resolution and local particle refinement}

A preliminary convergence study showed that the minimum spatial resolution to be adopted in these simulations should be $L / \Delta x_{\text {fine }}=400$ (used throughout this study unless mentioned), calling $L$ the helicopter length and $\Delta x_{\text {fine }}$ the finest inter-particle distance used in the vicinity of the body. This led to about $5 \times 10^{6}$ particles involved in the simulation. Furthermore, the number of particle neighbors is chosen so that $R / \Delta x=3$ everywhere in the discretized fluid domain, $R$ being the radius of the kernel compact support.

To reduce the number of particles (and therefore the computational costs), we used the adaptive particle refinement technique presented in Ref. [15]. Five particle refinement levels (i.e., five nested refinement boxes) are used here, leading to a spatial resolution $L / \Delta x_{\text {coarse }}=13$ in the coarsest zone. Note that the refinement boxes are "attached" to the body, so as to follow the helicopter during its computed movements. The position of these boxes is plotted in Fig. 6 .

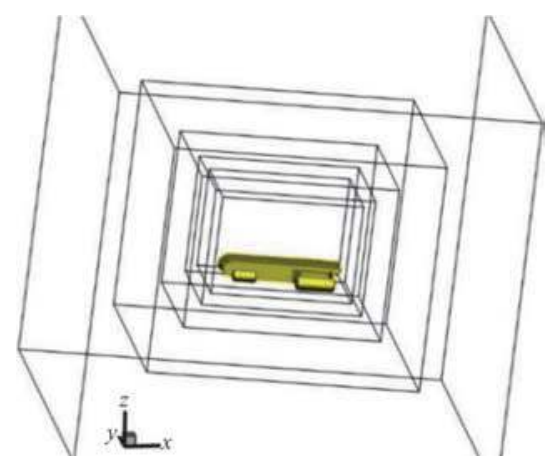

Fig. 6 (Color online) Initial view of the APR refinement boxes attached to the helicopter

\subsection{Flow separation on convex geometries water}

The violent impact of a body at the free surface is responsible for the generation of free surface jets, which should finally detach when running along a convex geometry. This phenomenon strongly affects the resulting flow depending on where and when the jet separates from the geometry, especially when the body is let freely in interaction with the fluid. Because of the complex nature of this phenomenon, possibly due to very local effects and depending certainly on several physical parameters, modelling properly the jet detachment in a single-phase and inviscid approach (so-called "non-viscous flow separation" ${ }^{\text {"[16]) }}$ ) is not obvious and has been discussed in several works. With the SPH method a special treatment is necessary to avoid such issues (see for instance ${ }^{[17]}$ ) as briefly illustrated in Fig. 7.
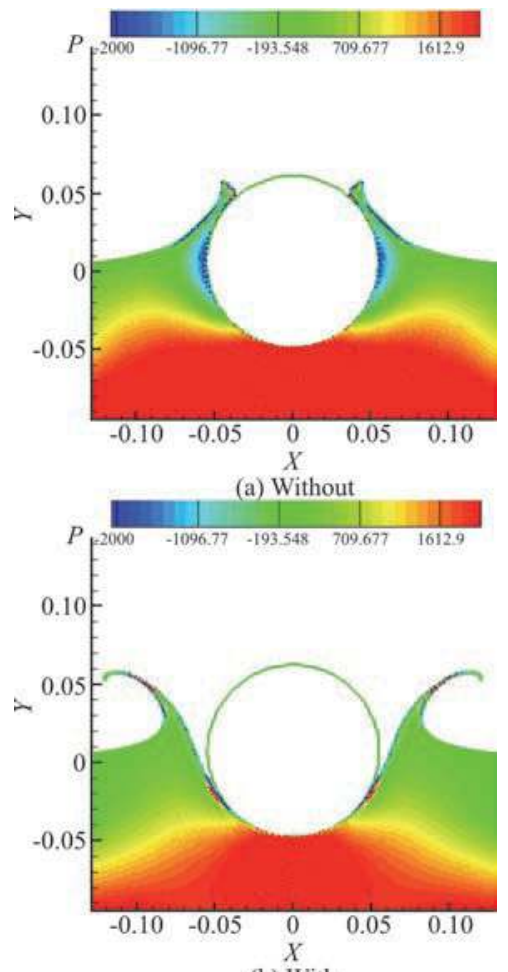

(b) With

Fig. 7 (Color online) Example of SPH simulation of a cylinder water entry flow separation technique $(t=0.025 \mathrm{~s})$

A specific technique inspired from Sun et al. ${ }^{[17]}$ has been therefore used in the present simulations, leading to significant effects on the helicopter behavior, as illustrated in Fig. 8 related to the impact case 1 detailed in Section 3.1.

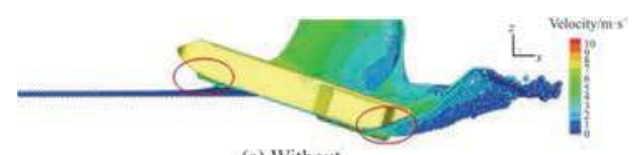

(a) Without

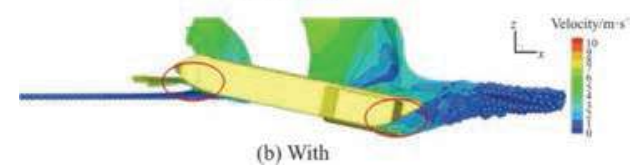

Fig. 8 (Color online) Effects of flow separation technique on the helicopter impact case

As visible in this figure, without the flow separation technique, the fluid jets stay attached both at the front and at the rear of the mock-up. Using the flow separation technique, the fluid jets detach at the front while maintaining physical suction effects at the rear, as the fluid stays attached to the body in this crucial area. 
This affects the pitch angle evolution, as shown in Fig. 9 where a first comparison with the experimental results underlines the importance of designing a proper flow separation technique.

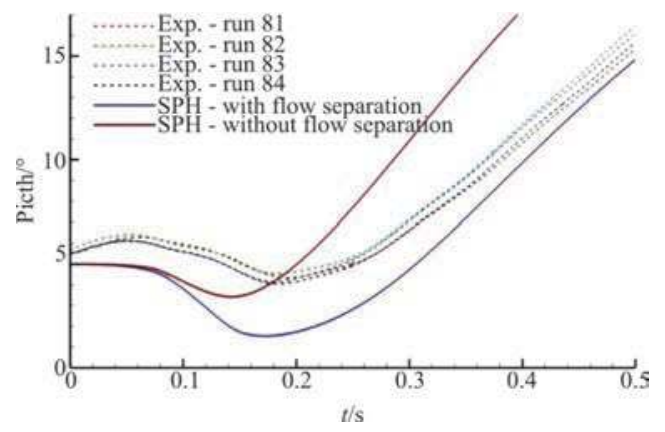

Fig. 9 (Color online) Effect of the flow separation technique on the pitch angle evolution (example on case 1 but with theoretical initial pitch angle $\theta_{0}=5.0^{\circ}$ ). Experimental results are also plotted for comparison purposes

\subsection{Modelling waves with SPH}

To account for the presence of waves in the numerical simulations, a coupling is made between the SPH and the high order spectral (HOS) ${ }^{[18]}$ method. The general principle of such a coupling is detailed in Ref. [2] and is schematized in Fig. 10.

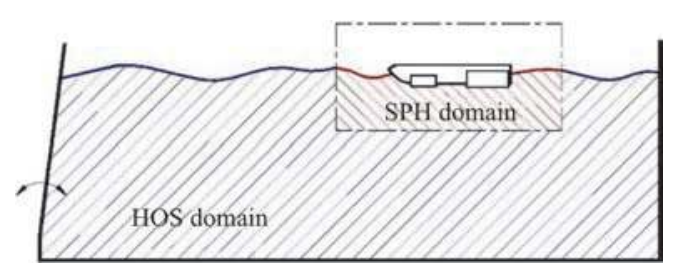

Fig. 10 (Color online) Schematic of the HOS/SPH coupling principle

It consists in decomposing the computational domain into two different ones: the HOS domain and the SPH domain. The present HOS-SPH algorithm is actually a one-way coupling, in the sense that the SPH solution uses the HOS wave data while no counterpart is provided from the SPH solution to the HOS waves. The incident HOS wave propagation solution is first computed (independently from SPH) for the full physical problem duration in order to reproduce accurately the experimental condition, and this solution is then entered as an input for SPH (the HOS solution is forced at the SPH domain boundaries through interpolations). This input consists in velocity and pressure maps on a regular grid covering the whole fluid domain at various instants (chosen with a sufficiently high frequency). This solution has been tested and successfully validated on complex naval cases $^{[2]}$, and is used in the present study for the case involving the presence of waves.

\section{Comparisons between numerical and experimen- tal results}

\subsection{Case 1: Impact on flat water}

The first case retained in the present study concerns the impact on flat water defined by the following conditions, measured at the impact instant: the velocity at location $G$ is $V x=-7.92 \mathrm{~m} / \mathrm{s}$, $V z=-0.81 \mathrm{~m} / \mathrm{s}$ (given in the global frame of reference $\{O, X, Y, Z\})$ and the initial pitch angle is $\theta_{0}=5.4^{\circ} \quad$ (value measured in the experiments).

The present work mainly focuses on first comparisons between numerical and experimental results. Firstly, a preliminary sensitivity study has been undertaken by varying the parameters that seem to play a crucial role in the helicopter behavior during its impact on flat water. Among the various parameters identified, the initial pitch angular velocity $\theta_{0}^{\prime}$ seems to be responsible for some significant effects on the helicopter response during the impact. A null pitch velocity was actually expected during the experiments. However, a value $\theta_{0}^{\prime} \approx 16^{\circ} / \mathrm{s}$ was observed. The origin of this effect is probably to be found in the way the mock-up exits from the rail guiding system, as the trolley wheels do not leave their respective rails exactly at the same instant, but this assumption still needs confirmation. Whatever the origin of this effect, we propose here to explore the effect of this initial pitch velocity on the resulting helicopter attitude during the impact. As a first investigation, Fig. 11 presents the pitch angle evolutions for $\theta_{0}^{\prime}=0^{\circ} / \mathrm{s}$ and $\theta_{0}^{\prime}=16^{\circ} / \mathrm{s}$, together with the results obtained for five different experimental runs.

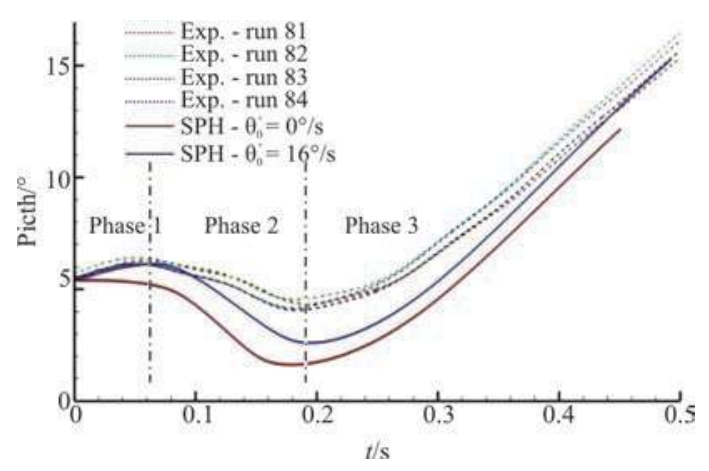

Fig. 11 (Color online) Impact Case 1: Effects of initial pitch angular velocity on the pitch angle evolution (with initial pitch angle taken as the experimental one, i.e., $\left.\theta_{0}=5.4^{\circ}\right)$ 
This figure illustrates the importance of the initial pitch angular velocity on the helicopter attitude. Note that the resulting pitch angle evolution strongly governs the behavior of the flow in interaction with the helicopter. Its evolution can be decomposed into three distinct phases, as depicted in Fig. 11. In phase 1, the pitch angle grows due to its initial angular velocity while the two rear floats enter slowly the free surface $(V z \cong 0.81 \mathrm{~m} / \mathrm{s})$, followed by the rear of the cabin. This phase mainly consists in a surfing impact, where suction effects together with the impact forces on the rear floats create a momentum which tends to decelerate the angular velocity, resulting in the decrease of the pitch angle observed in phase 2 . This decrease tends then to confine the free surface jet generated under the helicopter, due to its large horizontal velocity. The global pressure loads increase, together with the wetted surface, and the localization of the center of loads shifts from the rear to the front of the body. This finally results in an opposite momentum, leading to a strong increase of the pitch angle in phase 3 . This nose-down nose-up pitching motion was already observed in the simulations of Xiao et al. ${ }^{[9]}$.

The SPH simulation captures the global trends of the pitch angle evolution, especially when the experimental initial angular velocity is imposed. The main difference observed resides in phase 2 , where the numerical solution overestimates the decrease of the pitch angle (with a minimum angle of $2.5^{\circ}$ instead of $4^{\circ}$ in the experiments). This difference can be imputed to an overestimation of the positive momentum generated during the first instants of the impact, and is therefore due to the forces acting on the rear floats and at the rear of the cabin during phase 1 .

As a complementary analysis, the numerical and experimental evolutions of forces $F z$ along the $Z h$ axis on the three square plates $\mathrm{Sq} 1-\mathrm{Sq} 3$ are compared in Fig. 12. While the numerical force peaks tend to occur slightly later than the experimental ones for Sq3 and $\mathrm{Sq} 2$, it occurs a little bit earlier for $\mathrm{Sq} 1$ in the numerical solution. Indeed, since the pitch angle is smaller in the numerical solution than in the experiments, it leads to a faster displacement of the free surface jet under the helicopter cabin. This fact is also confirmed by the SPH solution with $\theta_{0}^{\prime}=0^{\circ} / \mathrm{s}$, for which the force peaks in Sq2 and Sq1 occur much earlier, due to the smaller pitch angle. For the same reason, while the amplitude of the experimental and numerical force peaks is in rather good agreement for $\mathrm{Sq} 3$ and $\mathrm{Sq} 2$, it is strongly overestimated for $\mathrm{Sq} 1$, and this difference is logically accentuated for the SPH solution with $\theta_{0}^{\prime}=0^{\circ} / \mathrm{s}$.

We draw the attention of the reader on the significant differences observed in the various experimental runs themselves, not so much in terms of maximum impact value but rather in time shift. The value of the shift correlates with the measured pitch at impact, underlining the sensitivity of these force evolutions with respect to this quantity. These differences are particularly visible for $\mathrm{Sq} 2$ and $\mathrm{Sq} 1$.

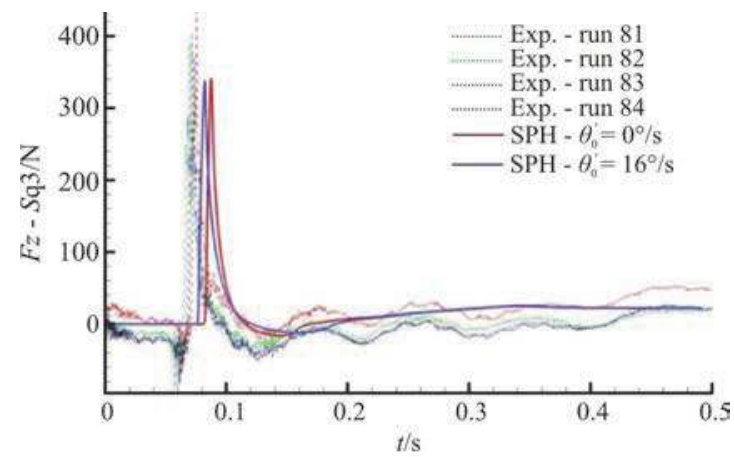

(a)

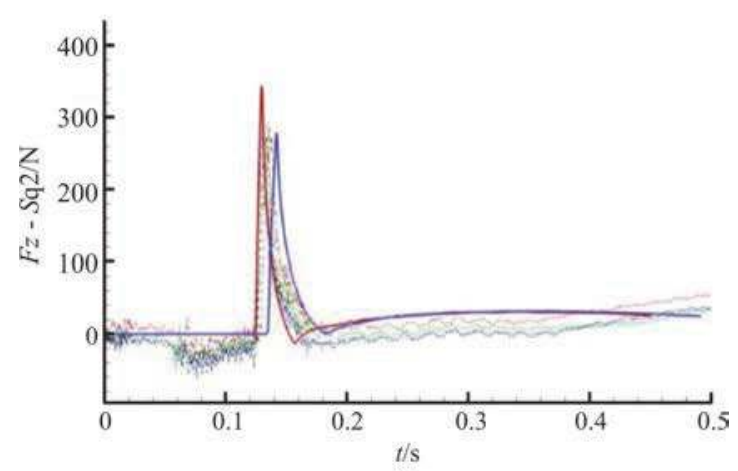

(b)

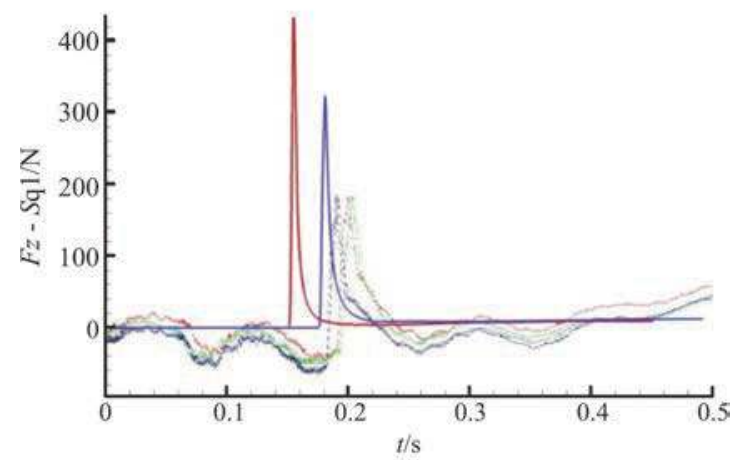

(c)

Fig. 12 (Color online) Impact case 1: Comparison of the numerical and experimental evolutions of forces $F z$ (along $Z h$ axis) on the three square plates $\mathrm{Sq} 3, \mathrm{Sq} 2$ and $\mathrm{Sq} 1$

For comparison purpose, Fig. 13 shows a qualitative comparison between the numerical and experimental free surface deformations.

A further simulation has been run again using an additional APR box to outline the importance of the spatial resolution, leading to $L / \Delta x_{\text {fine }}=800$ in the impact area. 


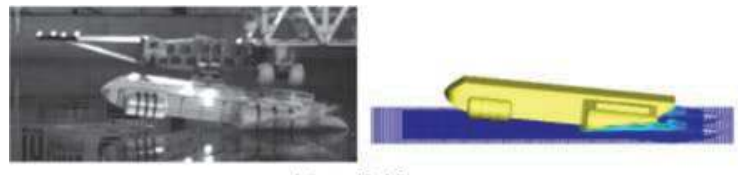

(a) $t=0.10 \mathrm{~s}$

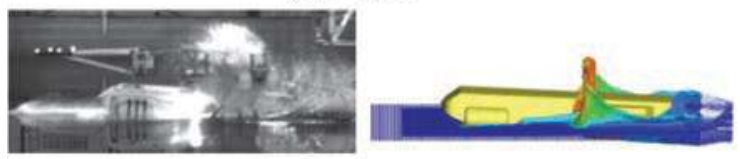

(b) $t=0.20 \mathrm{~s}$

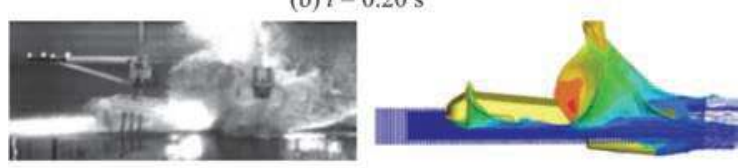

(c) $t=0.30 \mathrm{~s}$

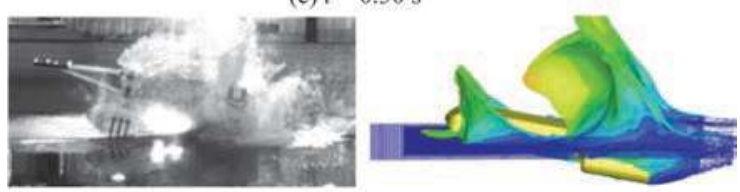

(d) $t=0.40 \mathrm{~s}$

Fig. 13 (Color online) Impact Case 1: Qualitative comparison between the experimental and numerical free surface deformations

As observed in Fig. 14, a significant improvement on the pitch angle evolution is observed, as also confirmed in Fig. 15 with the instant of occurrence and amplitude of the force peak on Sq1.

Note that the differences in the pitch angle are quite small at the very beginning of phase 2 , so that no significant differences are observed on $\mathrm{Sq} 2$ and $\mathrm{Sq} 3$, reason why their plots are omitted here.

Finally, when looking at the location of the separation points in Fig. 16, the improvements observed on the solution seem to be concentrated at the rear of the cabin, where strong suction effects occur.

\subsection{Case 2: Impact on waves}

The second impact case studied is much more challenging as it involves the presence of waves, characterized by a wavelength $\lambda=16.372 \mathrm{~m}$ and a wave height $H=0.94 \mathrm{~m}$, while the basin is $5 \mathrm{~m}$ deep. We define a phase offset between the mock-up CoG position with respect to the wave crest one at the impact instant such that, for a null phase $\varphi=0^{\circ}$, the $\mathrm{CoG}$ is located exactly upon the wave crest. This phase is taken here as $\varphi=196^{\circ}$, leading to an impact located around the wave trough as shown in Fig. 17. The velocity at location $G$ is $V x=-7.85 \mathrm{~m} / \mathrm{s}$, $V z=-3.88 \mathrm{~m} / \mathrm{s}$ and the initial pitch angle parameters are $\theta_{0}=10.7^{\circ}$ and $\theta_{0}^{\prime}=22.2^{\circ} / \mathrm{s} \quad$ (experimental values). An important feature of this case is its initial vertical velocity (much larger than in case 1), inducing theoretically larger loads onto the structure.

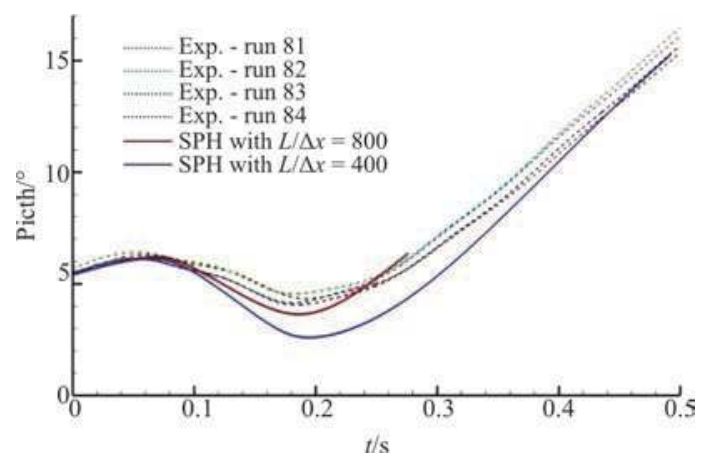

Fig. 14 (Color online) Impact Case 1: Effects of the spatial resolution on the pitch angle evolution (with $\varphi=5.4^{\circ}$ and $\theta_{0}^{\prime}=16.0^{\circ} / \mathrm{s}$ )

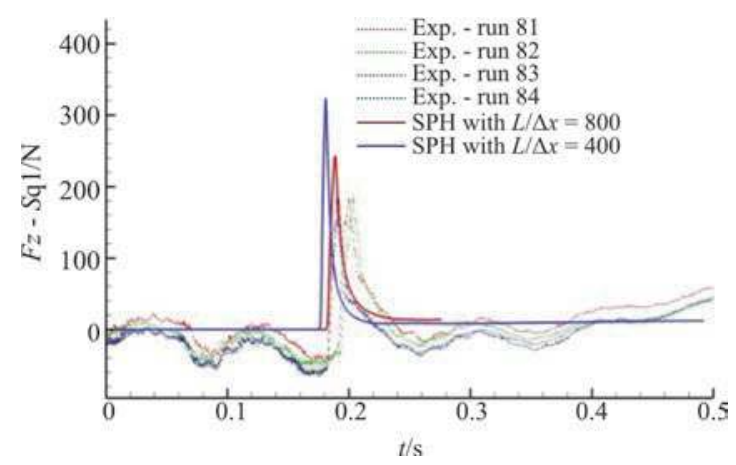

Fig. 15 (Color online) Impact Case 1: effect of increasing the spatial resolution on $S q 1$ (both with $\theta_{0}=5.4^{\circ}$ and $\left.\theta_{0}=16^{\circ} / \mathrm{s}\right)$

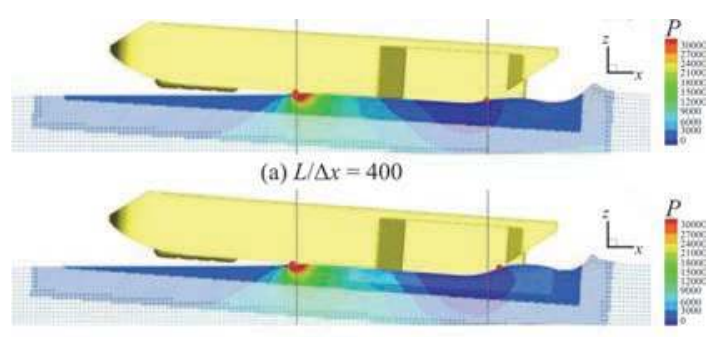

(b) $L / \Delta x=800$

Fig. 16 (Color online) Impact case 1: Effect of increasing the spatial resolution on the location of the separation points (red dots)

The same trend of evolution of the pitch angle (same three distinct phases) is observed in this impact case, as shown in Fig. 18 which compares the numerical and experimental solutions obtained.

As clearly visible in Fig. 19, the numerical force peaks at $\mathrm{Sq} 2$ and $\mathrm{Sq} 1$ occur earlier than in the experiments. The origin of this time difference seems to be due to an overestimation of the predicted jet velocity running under the cabin due to a smaller 


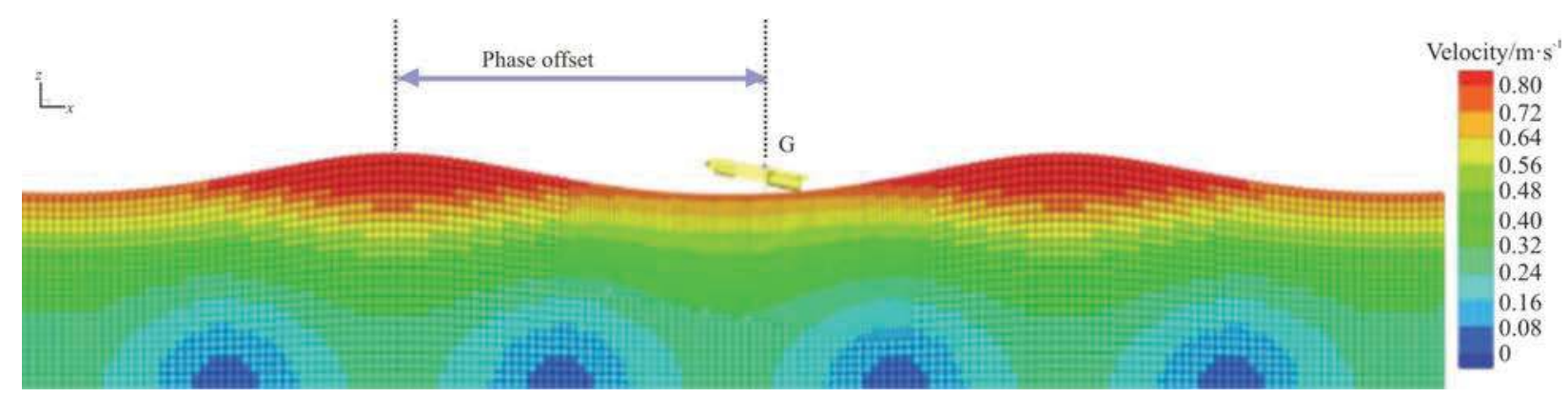

Fig. 17 (Color online) Impact case 2: Initial view of the helicopter location in waves

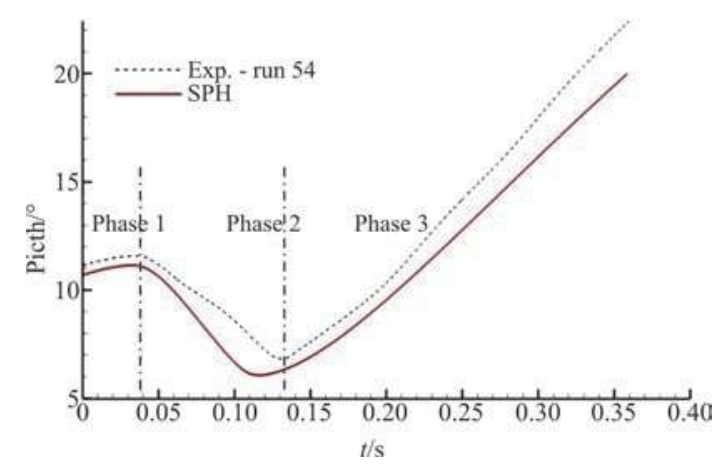

Fig. 18 (Color online) Impact case 2: comparison of the numerical and experimental evolutions of the pitch angle

numerical pitch angle than in the experiments, as already outlined for case 1 .

Furthermore, while the amplitude of these peaks is in good agreement for $\mathrm{Sq} 3$ and $\mathrm{Sq} 2$, a large overestimation of the amplitude is observed at Sq1, again because of the lower pitch angle predicted in the simulation.

Finally, a qualitative comparison between the numerical and experimental free surface deformations is proposed in Fig. 20.

\section{Conclusions}

In this paper, some challenging SPH simulations of helicopter ditching were presented and discussed on two distinct cases, considering the 6DOF of the body in coupled interaction with the fluid.

The first case studied involved a flat free surface together with a small initial pitch angle, a moderate vertical initial velocity and a large horizontal velocity. The second case involved the impact on waves, but this time with large initial pitch angle and vertical and horizontal velocity. The simulation results have been compared with the experimental results obtained at the wave basin of Ecole Centrale Nantes during the SARAH project. The experimental devices and settings retained in the present study have been first

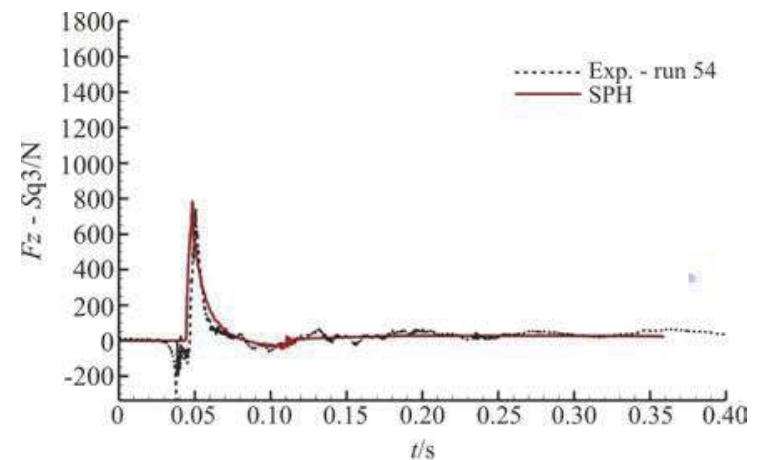

(a)

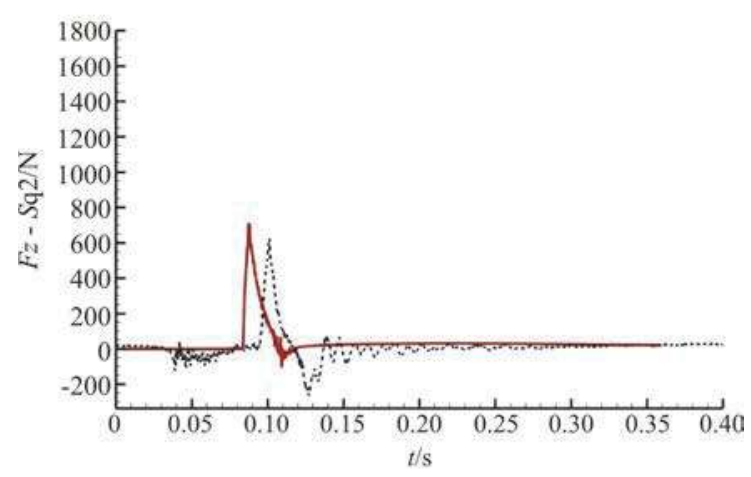

(b)

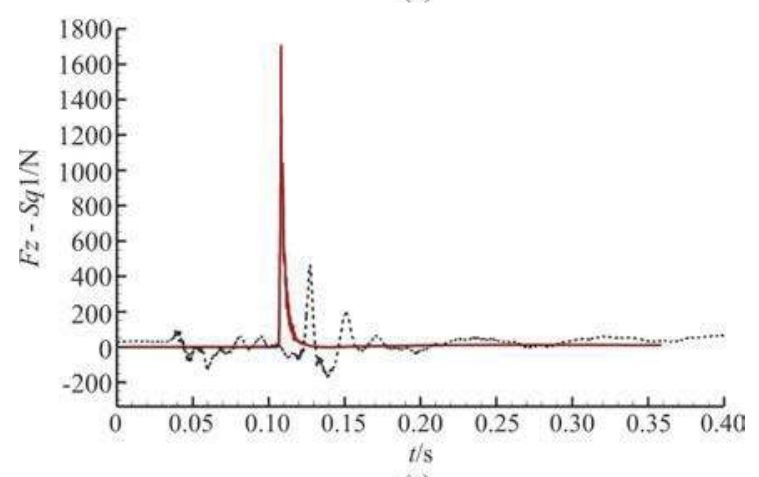

(c)

Fig. 19 (Color online) Impact case 2: Comparison of the numerical and experimental evolutions of forces $\mathrm{Fz}$ (along $\mathrm{Zh}$ axis) on the three square plates $\mathrm{Sq} 3, \mathrm{Sq} 2$ and $\mathrm{Sq} 1$ 


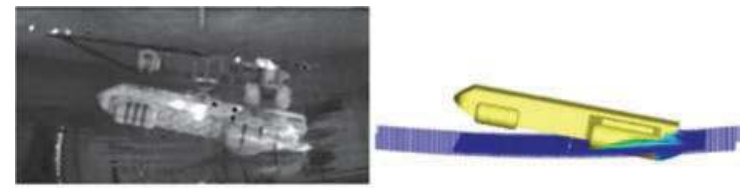

(a) $t=0.05 \mathrm{~s}$

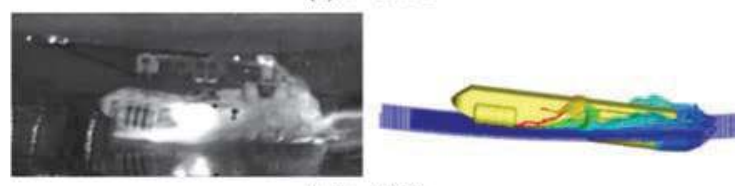

(b) $t=0.10 \mathrm{~s}$

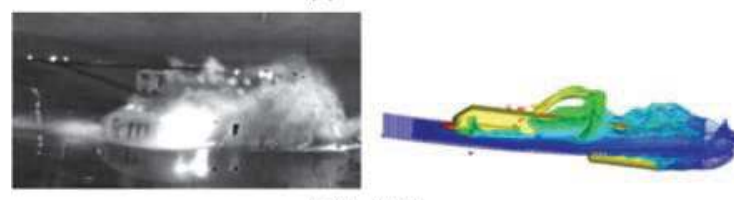

(c) $t=0.15 \mathrm{~s}$

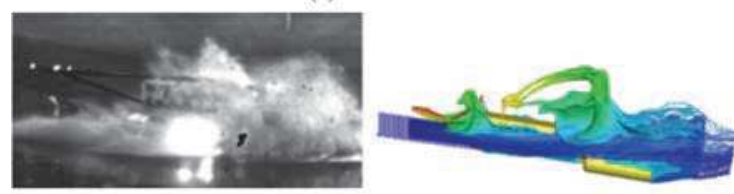

(d) $t=0.20 \mathrm{~s}$

Fig. 20 (Color online) Impact case 2: Qualitative comparison between the experimental and numerical free surface deformations

presented, followed by the SPH scheme and the functionalities needed to perform the simulations. In particular, the importance of using both Adaptive Particle Refinement and a flow separation technique has been outlined. Through these numerical simulations, for helicopter design purposes the importance of a proper capture of the pitch angle evolution together with considering its actual initial angular velocity have been emphasized. Despite the complexity of these impact cases, for which the experimental results themselves exhibited some deviations, encouraging numerical solutions have been obtained. The global trends of the pitch angle evolution have been retrieved, as well as the local impact forces under the helicopter cabin. The differences observed between the numerical and experimental pitch angle evolutions have proved to be at the origin of the resulting differences, visible namely through a possibly large overestimation of the local impact forces. Furthermore, the effects of the spatial resolution adopted was shown to be significant, in particular to capture properly the separation point at the rear of the cabin, where strong suction effects occur.

\section{Acknowledgements}

This work was supported by the European Union Horizon 2020 Research and Innovation Program
(Grant No. 724139). The authors thank the Pôle de Calcul et de Données Marines (PCDM) for providing DATARMOR storage and computational resources (http://www.ifremer.fr/pcdm), and the Institut de Calcul Intensif (ICI) (Grant No. 2018-P1804060).

\section{References}

[1] Bouscasse B., Ohana J., Abu Zarim M. et al. D5.2: Report on test data on helicopter ditching [R]. H2020-EU.3.4 Report, 2019.

[2] European Aviation Safety Agency-Certification Specifications for Small Rotorcraft CS-27 [S].

[3] European Aviation Safety Agency-Certification Specifications for Large Rotorcraft CS-29 [S].

[4] Oger G., Doring M., Alessandrini B. et al. Two-dimensional SPH simulations of wedge water entries [J]. Journal of Computational Physics, 2007, 213(2): 803-822.

[5] Oger G., Le Touzé D., Ducrozet G. et al. A coupled SPH-spectral method for the simulation of wave train impacts on a FPSO [C]. 33rd International Conference on Ocean Offshore and Arctic Engineering, San Francisco, USA, 2014.

[6] Pentecote N. Validation of PAM-CRASH code for the simulation of the impact on water [R]. Stuttgart, Germany: Institut für Bauweisen- und Konstruktionsforschung, DLR-IB 435.2003/3, 2003.

[7] Cartwright B. K., Chhor A., Groenenboom P. H. L. Numerical simulation of a helicopter ditching with emergency flotation devices [C]. 5th International SPHERIC workshop, Manchester, UK, 2010.

[8] Woodgate M. A., Barakos G. N., Scrase N. et al. Simulation of helicopter ditching using smoothed particle hydrodynamics [J]. Aerospace Science and Technology, 2019, 85: 277-292.

[9] Xiao T., Qin N., Lu Z. et al. Development of a smoothed particle hydrodynamics method and its application to aircraft ditching simulations [J]. Aerospace Science and Technology, 2017, 66: 28-43.

[10] Iafrati A., Grizzi S., Siemann M. H. et al. High-speed ditching of a flat plate: Experimental data and uncertainty assessment [J]. Journal of Fluids and Structures, 2015, 55: 501-525.

[11] Parshikov A. N., Medin S. A., Loukashenko I. I. et al. Improvements in SPH method by means of interparticle contact algorithm and analysis of perforation tests at moderate projectile velocities [J]. International Journal of Impact Engineering, 2000, 24(8): 779-796.

[12] Oger G., Marrone S., Le Touzé D. et al. SPH accuracy improvement through the combination of a quasiLagrangian shifting transport velocity and consistent ALE formalisms [J]. Journal of Computational Physics, 2016, 313: 76-98.

[13] Wendland H. Piecewise polynomial, positive definite and compactly supported radial functions of minimal degree [J]. Advances in Computational Mathematics, 1995, 4: 389-396.

[14] Chiron L., de Leffe M., Oger G. et al. Fast and accurate SPH modelling of 3D complex wall boundaries in viscous and non viscous flows [J]. Computer Physics Communications, 2019, 234: 93-111.

[15] Chiron L., Oger G., de Leffe M. et al. Analysis and improvements of adaptive particle refinement (APR) 
through CPU time, accuracy and robustness considerations [J]. Journal of Computational Physics, 2018, 354: 552-575.

[16] Sun H., Faltinsen O. M. Water impact of horizontal circular cylinders and cylindrical shells [J]. Applied Ocean Research, 2006, 28: 299-311.

[17] Sun P., Zhang A. M., Marrone S. et al. An accurate and efficient SPH modeling of the water entry of circular cylinders [J]. Applied Ocean Research, 2018, 72: 60-75.
[18] Ducrozet G., Bonnefoy F., Le Touzé D. et al. A modified high-order spectral method for wavemaker modeling in a numerical wave tank [J]. European Journal of Mechanics-B/Fluids, 2012, 34: 19-34. 